\title{
ALGEBRAIC MODEL OF DIFFERENCE EQUATIONS AND FUNCTIONAL EQUATIONS
}

\author{
JITKA LAITOCHOVÁ
}

\begin{abstract}
We will deal with the theory of Abel functional equations in the space of strictly monotonic functions $S$. The Abel functional equation model reduces under specialization to a linear functional or to a linear difference equation. Definitions, structure, and general theory for Abel functional equations on $S$ appear. The approach duplicates a rich body of known definitions, results and properties for classical functional and difference equations.

The setting for the algebraic model is in the space $S$ of strictly monotonic real functions $f$ defined on the interval $\mathcal{J}=(-\infty, \infty)$. It is required that $f$ map $\mathcal{J}$ one-to-one onto an interval $(a, b)$, where $a$ and $b$ are extended real numbers.

The model equation is expressed in terms of iteration of a function $\Phi$ in $S$. The iteration process uses a canonical function in $S$, which is an arbitrarily chosen increasing function $X \in S$.

A method is presented for solving the new model equation. This method can be applied to solve, in particular, some classical linear functional and difference equations.
\end{abstract}

\section{INTRODUCTION}

We will deal with the theory of Abel functional equations in the space of strictly monotonic functions. The first known model, introduced by O. Boruvka [1], is specific to differential equations, whereas the model we introduce is independent of differential equations. The Abel functional equation model reduces under specialization to a linear functional or a difference equation. The approach duplicates a rich body of known definitions, results and properties for classical functional and difference equations [3], [2], [8].

The setting for the algebraic model is in the space $S$ of strictly monotonic real functions $f$ defined on the interval $\mathcal{J}=(-\infty, \infty)$. It is required that $f$ map $\mathcal{J}$ one-to-one onto an interval $(a, b)$, where $a$ and $b$ are real or extended numbers.

The model equation is expressed in terms of iteration of a function $\Phi$ in $S$. The iteration process uses a canonical function in $S$, which is an arbitrarily chosen increasing function $X \in S$.

2010 Mathematics Subject Classification. 39B05; 39B12.

Key words and phrases. Linear $k$ th order functional equation, linear $k$ th order difference equation, space of continuous strictly monotonic functions, group multiplication, generalized Abel functional equation. 
The function $\Phi$ is algebraically a phase function known from Borùvka's theory of linear differential transformations of the second order [1]. The theory is useful for investigating oscillatory and asymptotic properties of solutions of ordinary linear differential equations. By a phase function, Boruvka meant a function $\alpha$ defined in an open interval $j$ such that $\alpha \in C_{1}, \alpha^{\prime} \neq 0$ for all $t \in j$. Each phase function $\alpha \in C_{3}$ represents, in its domain $j$, a first phase of the differential equation $y^{\prime \prime}=q(t) y$, where the first phase is any continuous function $\alpha$ defined on $j$ which satisfies $\tan \alpha(t)=\frac{u(t)}{v(t)}$ except at zeros of $v$, and $(u, v)$ is a solution basis of the equation.

A method is presented for solving the new model equation. This method can be applied to solve, in particular, some classical linear functional and difference equations.

Because difference equation theory uses the difference operator to express certain results, the notion of a difference operator is defined for Abel functional equations on $S$. Results are obtained for difference and sum operators, which reduce, when the model equation is a difference equation, to classical known results.

A solution of the linear functional equation is considered to be a continuous function $f$ on an interval $\left\langle x_{0}, \infty\right)$. A solution $f$ of the difference equation is considered on a sequence of points $\left\{x_{n}\right\}_{0}^{\infty}$.

If domains of coefficients and right sides of the equations are considered to be the interval $\mathcal{J}$, which is the union of intervals $j_{\mu}=\left(x_{\mu}, x_{\mu+1}\right), \mu=-\infty$ to $\infty$, then solutions of linear functional equations are continuous functions on $\mathcal{J}$ while solutions of linear difference equations are two-sided sequences $\left\{x_{n}\right\}_{n=-\infty}^{\infty}$. The definition of symbol $x_{n}$ appears below.

Algebraic models directly related to $\alpha(f(x))=\alpha(x)+1$, which is the classical Abel functional equation, are studied in [5]. The new models are treated abstractly as the (generalized) Abel functional equation $\alpha(f(x))=g(\alpha(x))$, in which $f$ and $g$ are given and $\alpha$ is the unknown. Given an increasing function $f$, possibly having fixed points in its domain $(a, b)$, a group-theoretic iterative explicit construction is given for infinitely many solutions $\alpha$ which are infinite at fixed points of $f$ and otherwise monotonic. The group-theoretic structure is suitable for studying solution properties of Abel functional equations. The methods apply in particular to Abel functional equations for which the domain $(a, b)$ is a finite interval, a halfline or the real line. The function $f$ is allowed to have many fixed points $\bar{x}$, defined by the equation $f(\bar{x})=X(\bar{x})$, where $X(x)$ is a canonical function in $S(X(x)=x$ gives classical fixed points).

It is shown how to form an Abel functional equation which represents a linear homogeneous functional equation with constant coefficients. Results of the specialization are stated. The Abel functional equation model in $S$ is able to simultaneously model both $\mathrm{k}$-th order linear difference equation with constant coefficients [4] and first order linear difference equations with constant and nonconstant coefficients [6]. Some applications appear, which show how to do the uniform modeling of classical equations. 


\section{SPACE OF STRICTLY MONOTONIC FUnCTIONS}

Definition 2.1: Given $a, b \in \bar{R}, a<b$. The set of all continuous functions on $(-\infty, \infty)$ which map one-to-one the interval $(-\infty, \infty)$ onto $(a, b)$ will be denoted by the symbol $S_{a}^{b}$ and called a space of strictly monotonic functions.

Definition 2.2: An arbitrarily chosen increasing function $X=X(x), X \in S$, will be called a canonical function in $S$. The inverse to the canonical function $X$ will be denoted by $X^{*}$.

Definition 2.3: Let $\alpha, \beta \in S$. The composite function $\gamma=\alpha\left(X^{*}(\beta(x))\right)$, shortly $\gamma=\alpha X^{*} \beta(x)$, will be called a product and denoted $\gamma=\alpha \circ \beta$.

Remark 2.1: The set $S$ with the operation of multiplication $\circ$ forms a noncommutative group.

Definition 2.4: Let $\phi \in S, \phi$ increasing and $X \in S$ be a canonical function, $\phi>X$ on $(-\infty, \infty)$. The iterates of $\phi$ in $S$ are given by

$$
\begin{gathered}
\phi^{0}(x)=X(x) \\
\phi^{n+1}(x)=\phi \circ \phi^{n}(x), \quad n=0,1,2, \ldots \\
\phi^{n-1}(x)=\phi^{-1} \circ \phi^{n}(x), \quad n=0,-1,-2, \ldots,
\end{gathered}
$$

$x \in(-\infty, \infty)$, where $\phi^{-1}$ is the inverse element according to $\circ$.

\section{Constant-COefficient $k$ Th ORDER EQuation}

Consider the homogeneous constant-coefficient equation

$$
a_{k} f \circ \phi^{k}(x)+a_{k-1} f \circ \phi^{k-1}(x)+\cdots+a_{0} f \circ \phi^{0}(x)=0,
$$

where $a_{i} \in R, i=0,1,2, \ldots, k$.

Functional Equation. If the $x$-domain is $(-\infty, \infty)$, then a solution of (HCCE) is a function $f \in C_{0}(-\infty, \infty)$ that satisfies the equation for all $x$.

Difference Equation. If the $x$-domain is a discrete sequence $\mathcal{O}=\left\{X^{*} \phi^{n}\left(x_{0}\right)\right\}$, then a solution of (HCCE) is a function $f$ defined on $\mathcal{O}$ which satisfies the equation at each $x=X^{*} \phi^{n}\left(x_{0}\right)$.

3.1. Generalized Abel functional equation. The solution $f(x)$ of (HCCE) will be given in terms of the generalized Abel equation

$$
\alpha \circ \phi(x)=g \circ \alpha(x) .
$$

We assume $g(x)=X(x+1)$, then the Abel equation is

$$
\alpha \circ \phi(x)=X(x+1) \circ \alpha(x) .
$$

Equation (AFE) is called the associated functional equation of (HCCE). Known are $X$ and $\phi$ in $S$. Function $\alpha$ is the solution of (AFE). 


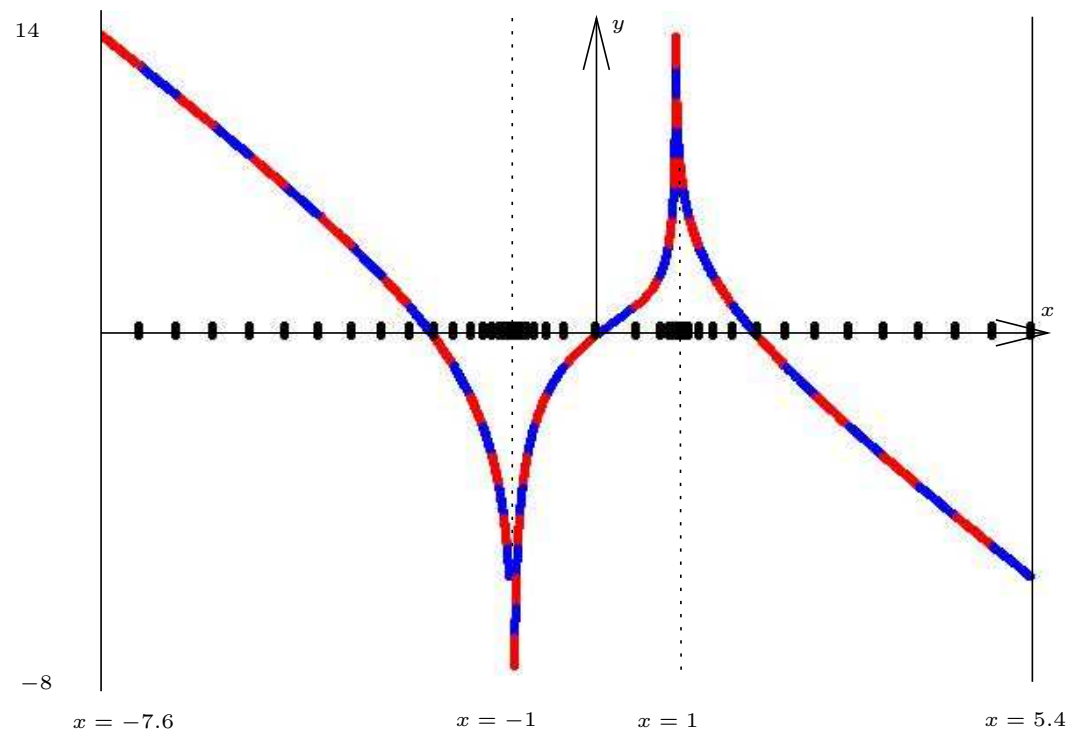

Figure. An iterative solution of $\alpha(\phi(x))=\alpha(x)+1$, where $\phi(x)=\frac{1}{1+x^{2}}-\frac{1}{2}+x$.

Example 3.1: We shall consider the specialized Abel functional equation

$$
\alpha(\phi(x))=\alpha(x)+1,
$$

where

$$
\phi(x)=\frac{1}{1+x^{2}}-\frac{1}{2}+x .
$$

The function $\phi$ has fixed points at $x= \pm 1$, where $\alpha$ is infinite. The fixed points separate regions of increase of $\alpha$ and decrease of $\alpha$. The segments of the solution $\alpha$ represent the iterative steps used to produce the graphic in the preceding figure. Generally, infinitely many iterations are required to fully represent $\alpha$.

For the theory of functional equations see [3]. The theory of difference equations can be found in [2] and [8]. For more results on generalized Abel functional equation and linear $k$ th order equations in $S$ see [4], [5], [6] and [7].

\subsection{Simple positive root theorem.}

Theorem 1. Let equation (HCCE) be given. Let $\lambda_{1}, \lambda_{2}, \ldots, \lambda_{k}$ be simple positive roots of the characteristic equation

$$
a_{k} \lambda^{k}+a_{k-1} \lambda^{k-1}+\cdots+a_{1} \lambda+a_{0}=0
$$

Let $\alpha$ be a continuous solution of the associated Abel functional equation (AFE).

Then the functions

$$
f_{1}=\lambda_{1}^{X^{*} \alpha(x)}, f_{2}=\lambda_{2}^{X^{*} \alpha(x)}, \ldots, f_{k}=\lambda_{k}^{X^{*} \alpha(x)},
$$

are linearly independent solutions of (HCCE). 


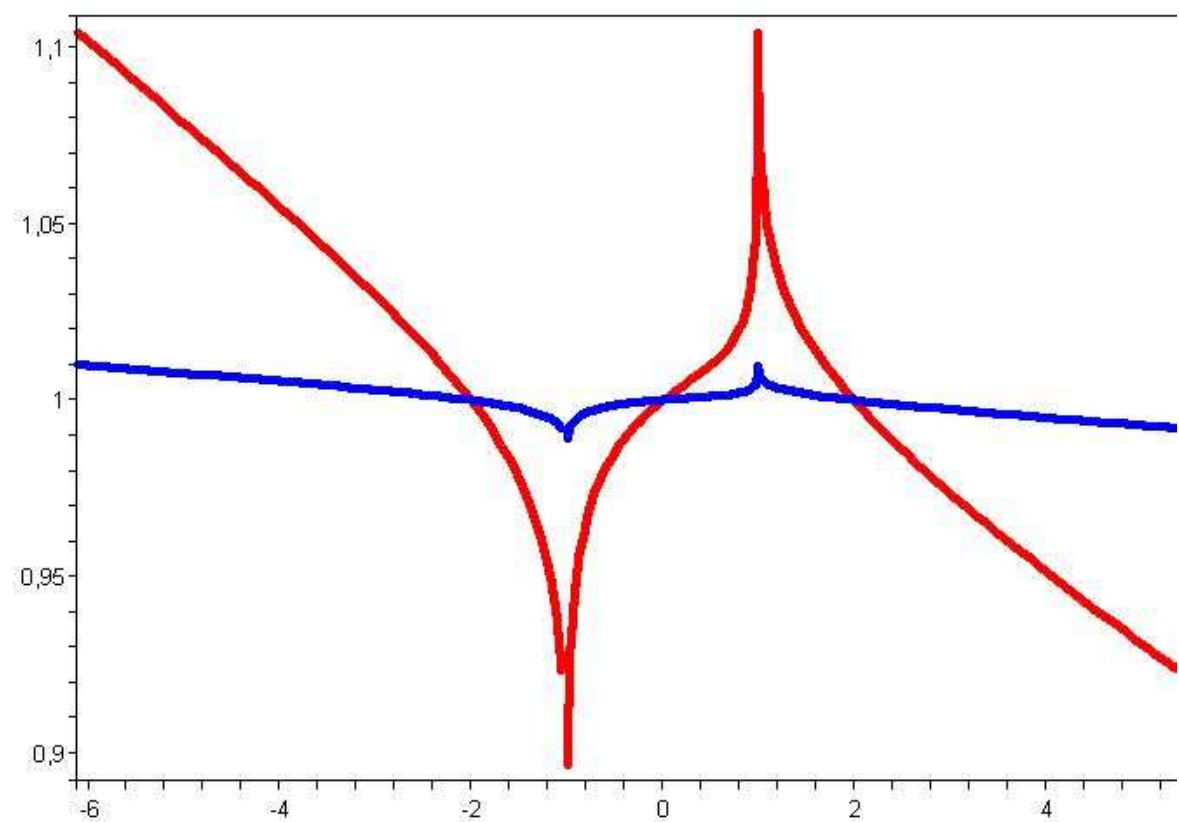

Figure. Iterative solutions $f_{1}(x)=1.01^{\alpha(x)}, \quad f_{2}(x)=1.001^{\alpha(x)}$.

Example 3.2: The second order functional equation

$$
f(\phi(\phi(x)))-2.011 f(\phi(x))+1.01101 f(x)=0
$$

with

has solutions

$$
\phi(t)=\frac{1}{1+t^{2}}-\frac{1}{2}+t
$$

$$
f_{1}(x)=1.01^{\alpha(x)}, \quad f_{2}(x)=1.001^{\alpha(x)} .
$$

The Abel solution $\alpha$ is obtained by iteration on three intervals between fixed points of $\phi$, to obtain the following graphical representation of $f_{1}, f_{2}$. See the preceding figure.

3.2.1. A difference equation application. The classical difference equation is a specialization of functional equation (HCCE). The space $S$ is the set of all monotonic continuous $\phi$ mapping $(-\infty, \infty)$ onto itself. Let's assume

$$
X(x)=x \text { (identity), } \quad X^{*}(x)=x, \quad \phi(x)=x+1 .
$$

Then the Abel functional equation is

$$
\alpha(x+1)=\alpha(x)+1
$$

and it has a solution $\alpha(x)=x$.

The corresponding constant-coefficient linear homogeneous equation (HCCE) specializes to an equation of the form

$$
a_{k} f(x+k)+a_{k-1} f(x+k-1)+\cdots+a_{0} f(x)=0 .
$$


If $\lambda>0$ is a root of the characteristic equation, then a special solution is $f(x)=\lambda^{X^{*} \alpha(x)}$, where $\alpha$ satisfies the Abel equation. Because $\alpha(x)=x$ and $X(x)=x$, then the special solution is

$$
f(x)=\lambda^{x} .
$$

\subsection{Multiple positive root theorem.}

Theorem 2. Let constant-coefficient equation (HCCE) be given. Let $\lambda_{0}$ be a positive real root of the characteristic equation

$$
a_{k} \lambda^{k}+a_{k-1} \lambda^{k-1}+\cdots+a_{1} \lambda+a_{0}=0
$$

of multiplicity $s, 1 \leq s \leq k$. Let $\alpha(x)$ be a continuous solution of Abel functional equation (AFE) and let $X^{*}$ be the inverse function to canonical function $X$.

Then the functions

$$
f_{r}(x)=\left(X^{*} \alpha(x)\right)^{r} \lambda_{0}^{X^{*} \alpha(x)}, \quad 0 \leq r<s
$$

are independent solutions of equation (HCCE).

Example 3.3: The second order functional equation

$$
f(\phi(\phi(x)))-3 f(\phi(x))+2.25 f(x)=0 \quad \text { with } \quad \phi(t)=\frac{1}{1+t^{2}}-\frac{1}{2}+t
$$

has solutions

$$
f_{1}(x)=1.5^{\alpha(x)}, \quad f_{2}(x)=1.5^{\alpha(x)} \alpha(x) .
$$

The Abel solution $\alpha$ is obtained by iteration on three intervals between fixed points of $\phi$, to obtain a graphical representation of $f_{1}, f_{2}$. 


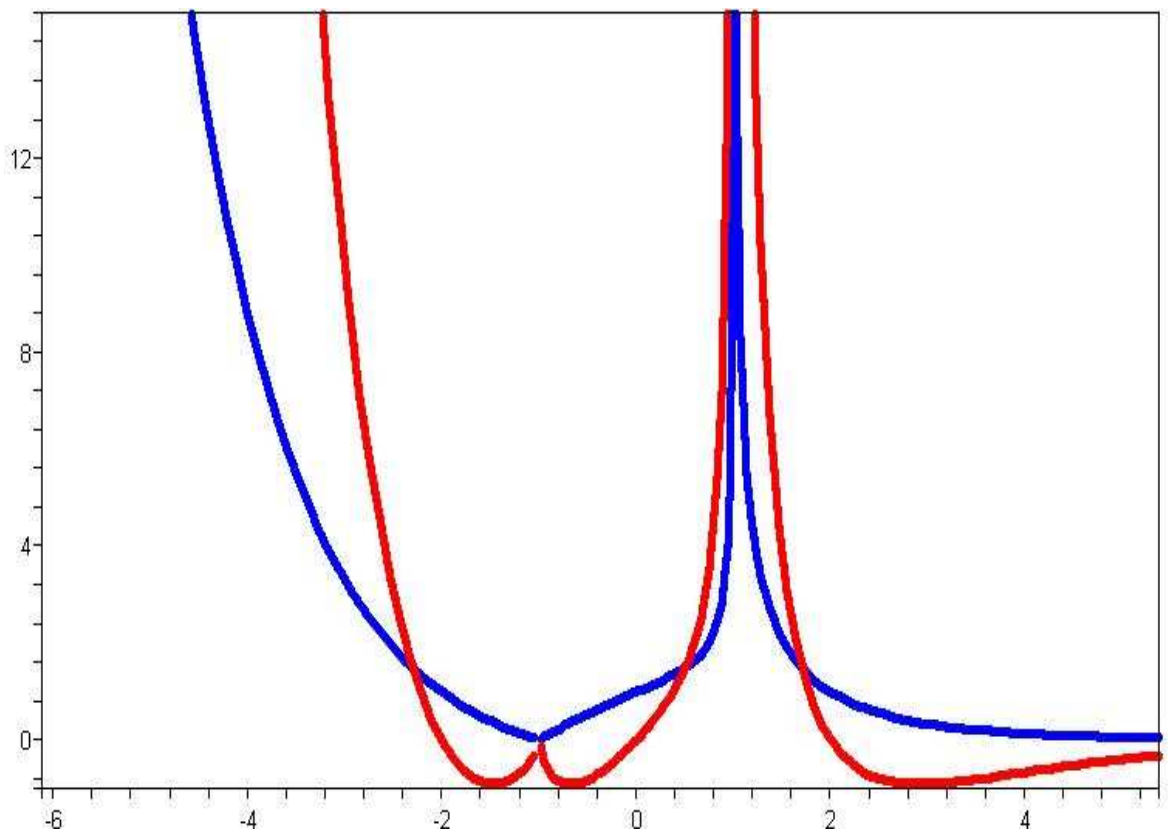

Figure. Iterative solutions $f_{1}=1.5^{\alpha(x)}$ and $f_{2}=1.5^{\alpha(x)} \alpha(x)$.

Example 3.4 (Difference Equation: Double Positive Real Root): The second order functional equation

$$
f(\phi(\phi(x)))-3 f(\phi(x))+2.25 f(x)=0 \quad \text { with } \quad \phi(t)=\frac{1}{1+t^{2}}-\frac{1}{2}+t,
$$

can be specialized to a difference equation on the domain $\mathcal{O}=\left\{\phi^{n}\left(x_{0}\right)\right\}$. The solutions

$$
f_{1}(x)=1.5^{\alpha(x)}, \quad f_{2}(x)=1.5^{\alpha(x)} \alpha(x)
$$

obtained for the case $(-\infty, \infty)$ give rise to difference equations solutions on the intervals between fixed points of $\phi$.

The Abel solution $\alpha$ is only required at points of $\mathcal{O}$. Iteration at starting values $x_{0}=-2,0,2$ gives the following graphical representation of $f_{1}, f_{2}$. Circles represent the discrete solution. 


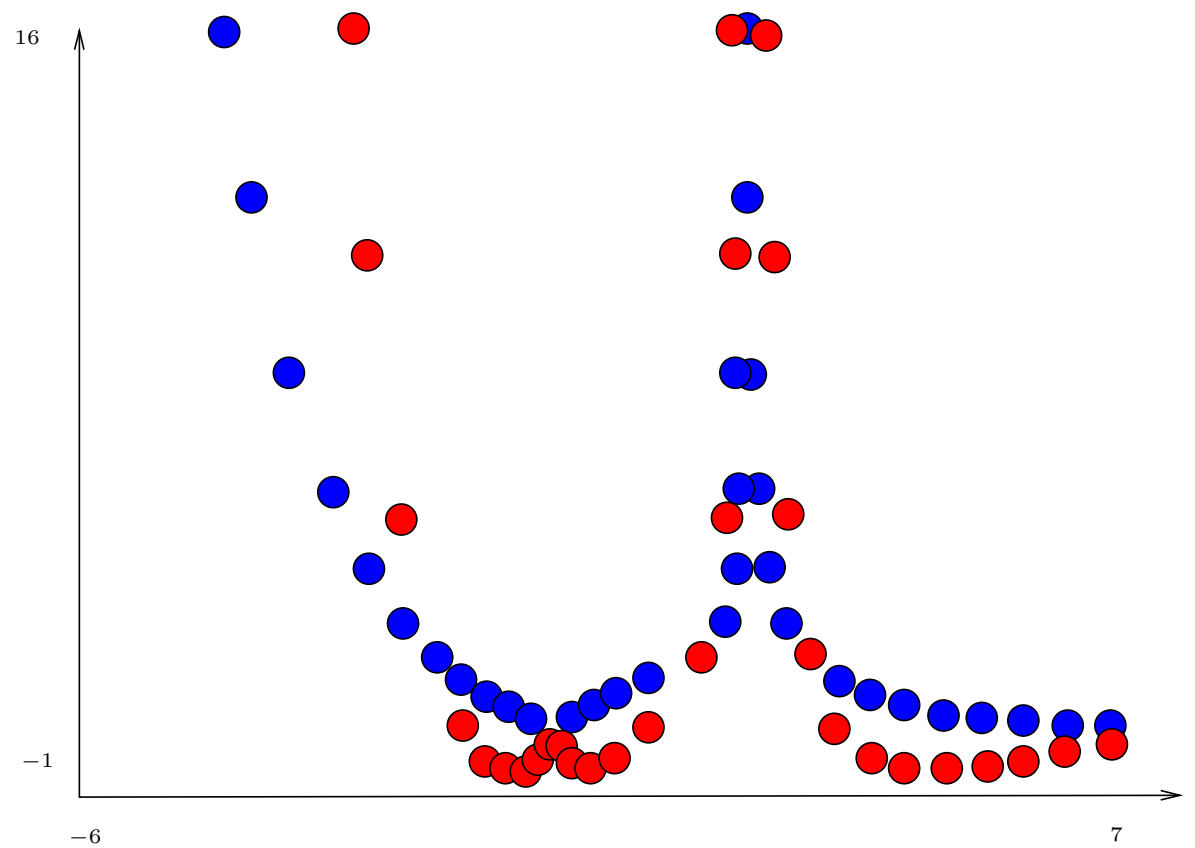

Figure. Iterative solutions $y=f(x)$ for $f(\phi(\phi(x)))-3 f(\phi(x))+2.25 f(x)=0$.

\subsection{Conjugate Root Theorem.}

Theorem 3. If the characteristic equation

$$
a_{k} \lambda^{k}+a_{k-1} \lambda^{k-1}+\cdots+a_{1} \lambda+a_{0}=0
$$

has conjugate complex roots

$$
\lambda_{1}=\bar{\lambda}_{2}=r(\cos \omega+i \sin \omega),
$$

then the corresponding linear homogeneous functional equation possesses two solutions in the form

$$
\begin{aligned}
& f_{1}=r^{X^{*} \alpha(x)} \cos \left(\omega X^{*} \alpha(x)\right), \\
& f_{2}=r^{X^{*} \alpha(x)} \sin \left(\omega X^{*} \alpha(x)\right) .
\end{aligned}
$$

Proofs of the above theorems can be found in [4].

Example 3.5: $\mathrm{x}$

Consider the second order functional equation

$$
f(\phi(\phi(x)))+f(x)=0 \quad \text { with } \quad \phi(t)=\frac{1}{1+t^{2}}-\frac{1}{2}+t .
$$

Two solutions are

$$
f_{1}=\cos \left(\frac{\pi}{2} \alpha(x)\right), \quad f_{2}=\sin \left(\frac{\pi}{2} \alpha(x)\right) .
$$

The iterative Abel solution $\alpha$ gives a graphical representation of $f_{1}, f_{2}$. 


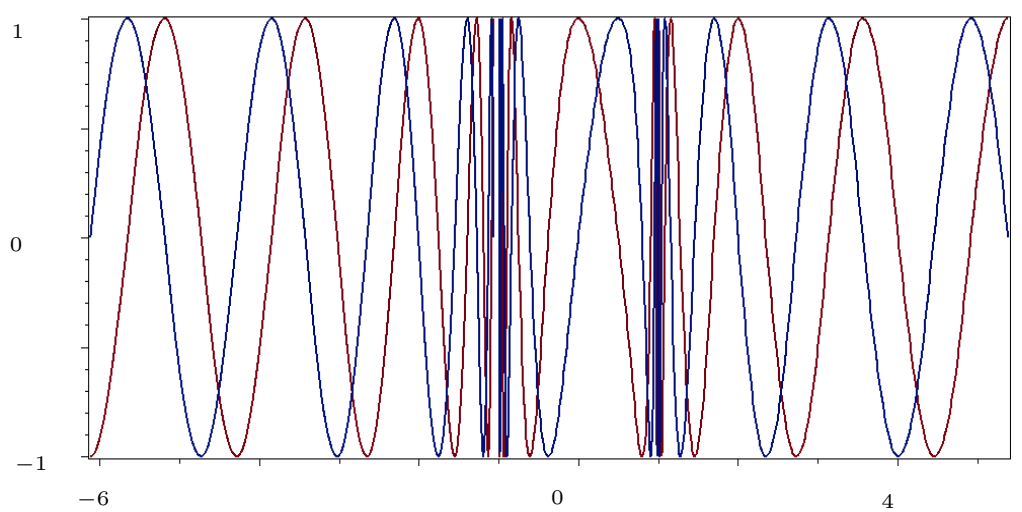

Figure. Iterative solutions $f_{1}=\cos \left(\frac{\pi}{2} \alpha(x)\right), f_{2}=\sin \left(\frac{\pi}{2} \alpha(x)\right)$.

Acknowledgements. This publication was supported by project IGA PdF 2014 026 Student Grant Competition of Palacký University in Olomouc.

\section{REFERENCES}

[1] O. Borùvka, Linear Differential Transformations of the Second Order, The English University Press, London, 1971.

[2] S. N. Elaydi, An Introduction to Difference Equations, Springer, New York, Berlin, Heidelberg, 1999 .

[3] M. Kuczma, B. Choczewski and R. Ger, Iterative Functional Equations, Cambridge University Press, Cambridge, Warszawa, 1990.

[4] J. Laitochová, A remark on $k$-th order linear functional equations with constant coefficients, Advances in Difference Equations, vol. 2006, Article ID 72615, 8 pages (2006).

[5] J. Laitochová, Group iteration for Abel's functional equation, Nonlinear Analysis: Hybrid systems 1 (2007), 95-102.

[6] J. Laitochová, Linear difference operators in the space of strictly monotonic functions, Tatra Mt. Math. Publ. 38 (2007), 111-121.

[7] J. Laitochová, Solution structure for homogeneous linear functional equations in $S$, Folia FSN Universitatis Masarykianae Brunensis, Mathematica 16, CDDE, Brno 2006, 123-129.

[8] A. Prágerová, Diferenční rovnice, SNTL Práce Praha, 1971.

Department of Mathematics, Faculty of Education, Palacký University, Olomouc, Czech Republic

E-mail address: jitka.laitochova@upol.cz 